\title{
Estrategias para la conservación del ecosistema páramo en Pulinguí San Pablo y Chorrera Mirador, Ecuador
}

\section{(Strategies for conservation of highland ecosystem in Pulinguí San Pablo and Chorrera Mirador, Ecuador)}

\author{
Patricio Lozano ${ }^{1}$, Aracely Armas ${ }^{1}$, Verónica Machado ${ }^{1}$
}

\begin{abstract}
Resumen:
Impulsar procesos de conservación y uso sustentable de un ecosistema implica en primera instancia, el entendimiento del territorio como un socio-ecosistema, y en segunda instancia, la aplicación del enfoque ecosistémico. Por este motivo, la presente es una herramienta que contribuye al manejo del ecosistema páramo de las comunidades de Pulinguí San Pablo y Chorrera Mirador. Este proceso consistió en la caracterización del socio-ecosistema, la determinación de objetos de conservación, y la formulación de estrategias para la conservación y uso sustentable. Los datos indican que en este socio-ecosistema existen 186 habitantes, que se benefician principalmente de tres tipos de servicios ecosistémicos (aprovisionamiento, regulación y culturales), y que se dedican a actividades agropecuarias y turísticas. Estos asentamientos ejercen en el socio-ecosistema una presión antrópica moderada, por cuanto, apenas 98.65 ha (de 4567.2 ha) están dedicadas a estas actividades. Esto a su vez se refleja en el estado de los objetos de conservación identificados, que evaluados a partir de tamaño, condición y contexto paisajístico, obtuvieron un promedio de 3,3/4,0 (puntos) que significa que se encuentran en proceso de deterioro; sin embargo, sus impactos se los puede minimizar con ocho estrategias construidas y ejecutadas por las poblaciones locales.
\end{abstract}

Palabras clave: socio-ecosistema; enfoque ecosistémico; objetos de conservación; amenazas.

\begin{abstract}
:
To drive conservation processes and sustainable use of an ecosystem implies, on the one hand, the understanding of the territory as a socio-ecosystem, and on the other hand, the application of the ecosystemic approach. For this reason, this is a tool that contributes to the highland ecosystem management of the communities Pulinguí San Pablo and Chorrera Mirador. This process was based on the socio-ecosystem characterization, determination of conservation elements and formulation of conservation and use of sustainable strategies. Data show that 186 habitants live in these socio-ecosystem and mainly benefit from three types of ecosystemic services: supply, regulation and cultural, carrying out agricultural, livestock and touristic activities. These settlements exert a moderated anthropic pressure in the socioecosystem due to the fact that only 98,65 hectares (out of 4567,20 hectares) are used for these activities. This is reflected in the state of the identified conservation elements that, evaluated according to their size, condition and landscaping context, obtained a mean of 3,3/4,0 (points) which means that they are in a decay process, nonetheless, their impacts may be diminished using established and executed strategies by local populations.
\end{abstract}

Keywords: socio ecosystem; ecosystemic approach; conservation objects; threads.

\footnotetext{
${ }^{1}$ Escuela Superior Politécnica de Chimborazo, Riobamba - Ecuador (plozano@espoch.edu.ec)
} 


\section{Introducción}

Estudiar las relaciones entre naturaleza y sociedad implica analizar la relación en doble vía, es decir, cómo el ser humano afecta a la integridad de los ecosistemas y cómo estos repercuten en el bienestar humano (Martín-López, Gómez, \& Montes, 2009). Esta visión instrumental de los ecosistemas se ha revalorizado en el marco conceptual para la Evaluación de Ecosistemas del Milenio, en donde se pone de manifiesto las estrechas relaciones entre la biodiversidad, el funcionamiento de los ecosistemas, el suministro de servicios ecosistémicos y el bienestar humano (Millenium Ecosystem Assessment, 2003, Camacho \& Ruiz, 2011). En otras palabras, se constituye un socio-ecosistema, por cuanto, en un proceso de coevolución; los sistemas humanos y los ecosistemas se han ido moldeando y adaptando conjuntamente, convirtiéndose en un sistema integrado de humanos en la naturaleza (Anderies, Janssen, \& Ostrom, 2004, Montes \& Sala, 2007). Por tanto, para gestionar estos ecosistemas es necesaria la aplicación del enfoque ecosistémico, entendido como una estrategia para el manejo integrado de los recursos que promueva la conservación y uso sustentable de forma justa y equitativa (Shepherd, 2006).

Los páramos son ecosistemas frágiles dominados por una vegetación herbácea y arbustiva; se ubican sobre los 3.000 y 5.000 msnm (entre el bosque cerrado y nieves perpetuas) a lo largo de los Andes húmedos entre Perú, Ecuador, Colombia y Venezuela, con extensiones hasta Costa Rica y Panamá (Hosftede, et al, 2014). En el Ecuador, el páramo cubre un $6 \%$ del territorio nacional (alrededor de 1.250 .000 ha) distribuido en comunidades indígenas (40\%), áreas protegidas (40\%), y haciendas (20\%), donde viven unas 500.000 personas (Mena, Medina, \& Hosftede, 2001). Este ecosistema en la provincia de Chimborazo cubre una extensión del 36,9\% y al igual que en los páramos del país se caracteriza por su gran diversidad de fuentes de agua dulce y por contener una alta capa de materia orgánica (Beltrán, 2010). Además aquí se asientan poblaciones que aprovechan los recursos de estas zonas para su subsistencia, como es el caso de la parroquia San Juan, una de las diez parroquias rurales del cantón Riobamba, constituida por 5 barrios y 28 comunidades. Una sección de esta parroquia forma parte de la Reserva de Producción de Fauna Chimborazo y cuenta con potencialidades naturales y culturales que se constituyen en una estrategia importante para el desarrollo sostenible de la localidad (Armas, 2014).

Adicionalmente, en esta provincia, las áreas intervenidas, tanto para agricultura como ganadería intensiva y extensiva, representan más del $74 \%$ frente a apenas $18 \%$ de ecosistemas en estado natural (Albán, Argüello, \& Bustamante, 2011), situación que no es ajena al páramo de las comunidades de Pulinguí San Pablo y Chorrera Mirador, por cuanto, la estructura, composición, y función del mismo, están siendo afectadas por presiones antrópicas que ejercen los asentamientos humanos a través de la conversión del uso del suelo, avance de la frontera 
agropecuaria, quema de páramo y pérdida de conocimientos tradicionales de conservación del ecosistema. Ante esta situación los habitantes de las referidas comunidades requieren construir actuaciones que contribuyan a la conservación y uso sustentable de sus recursos.

Para atender la situación descrita en las referidas comunidades, el presente trabajo planteó tres preguntas de investigación: ¿cuáles son las características del socio-ecosistema páramo?, ¿cuáles son los objetos de conservación del socio-ecosistema páramo?, y ¿qué estrategias se deberían impulsar para la conservación del socio-ecosistema páramo? Para resolver estas preguntas se formularon tres objetivos: a) caracterizar el socio- ecosistema del páramo, b) determinar objetos de conservación del socio-ecosistema páramo, c) formular estrategias para la conservación del socio-ecosistema páramo. En este contexto el propósito de esta investigación es la formulación participativa de una propuesta que contribuya a la conservación y uso sustentable del páramo, de tal forma que los servicios generados en el socio- ecosistema aporten al bienestar humano en todos sus componentes (Hofstede, 2008).

\section{Metodología}

\section{1. Área de Estudio}

Las comunidades de Chorrera Mirador (X 750008, Y 9827175, Z 4072msnm) y Pulinguí San Pablo ( $X$ 751694, Y 982617, Z 3910msnm) se ubican en la denominada zona alta de la parroquia San Juan (GAD Parroquia-Rural de San Juan, 2011), a 16,98 km de la cabecera parroquial y 34,98 km de la ciudad de Riobamba en la provincia de Chimborazo. Para acceder a estas comunidades se cuenta con vías de primer orden (Vía Riobamba- Guaranda).

En cuanto a la división política, Pulinguí San Pablo limita al norte con la parroquia San Andrés, al sur con la Comunidad Chimborazo, al este la Comunidad Chorrera Mirador, al oeste la Comunidad Ganquis, provincia de Bolívar. Chorrera Mirador limita al norte con la provincia de Bolívar, al sur con la Comunidad Cooperativa Santa Teresita, Comunidad Chinigua, al este con la Comunidad Cooperativa Santa Teresita, y al oeste con la Comunidad Pulinguí San Pablo. La parte alta de estas comunidades se encuentra en la Reserva de Producción de Fauna Chimborazo donde se pueden encontrar tres tipos de vegetación: herbácea, seco, gelidofitia (Sierra, 1999); alcanza una extensión total de 4567,2 ha, donde el 97,84\% (4468,7 ha) está cubierto en su mayoría por Calamagostris, Stipa y pequeños relictos de Azorella (GAD parroquia-Rural de San Juan, 2011). 


\subsection{Métodos}

La presente investigación midió de forma independiente las variables e indicadores que permitieron caracterizar, comprender, y analizar el socio-ecosistema de páramo, y a su vez, formular estrategias para su conservación. Para cumplir este propósito se aplicaron métodos y técnicas de investigación de campo y documental, las cuales se describen por objetivo.

\subsubsection{Caracterización del socio-ecosistema páramo}

El socio-ecosistema se caracterizó aplicando dos métodos: 1) el método de investigación documental, la técnica de síntesis, y las orientaciones de estructuración de información propuestas por (CONOGOPARE, 2011). La recolección de datos se realizó del Plan de Desarrollo y Ordenamiento Territorial de la parroquia San Juan (GAD parroquia-Rural de San Juan, 2011); 2) el método de investigación de campo, las técnicas de mapeo de servicios y oportunidades, mapeo de recursos naturales y uso de la tierra, diálogo semiestructurado para grupos focales y muestreo de calidad de agua, y las orientaciones de estructuración de información propuestas por (SENPLADES, 2011). La recolección de datos se hizo a través de talleres participativos desarrollados con los habitantes de la comunidad de Pulinguí San Pablo y Chorrera Mirador, y por medio de visitas in-situ a las vertientes de agua del área de estudio. Los resultados se presentaron en los ámbitos: ambiental, económico, sociocultural, y político-administrativo.

\subsubsection{Determinación de objetos de conservación del socio-ecosistema páramo}

Los objetos de conservación se determinaron aplicando el método de investigación de campo, la técnica de observación directa, las orientaciones para desarrollar el análisis valorativo de objetos de conservación propuesto por (Granizo, et al, 2006) y la clasificación de vegetación propuesta por (Sierra, 1999; Ministerio del Ambiente, 2012, 2014). La recolección de datos se realizó en los sitios de muestreo: prácticas productivas ancestrales ( $X$ 740501, Y 983045, Z 3838 msnm), páramo herbáceo (X 9830455, Y 9830916, Z 3937 msnm), páramo seco (X 741988, Y 9834408, Z 4671 msnm), Gelidofitia (X 739805 Y 9837044), y microcuenca Río Chimborazo (X 741355, Y 9830201, Z 3748 msnm). Los resultados de objetos de conservación se presentaron considerando las categorías natural y cultural.

\subsubsection{Formulación de estrategias de conservación para el socio-ecosistema páramo}

Las estrategias de conservación se formularon aplicando el método de investigación de campo, la técnica de diagramación de amenazas-estrategias, las orientaciones para desarrollar el análisis valorativo de amenazas de objetos de conservación propuesto por (Granizo, et al, 2006) y los lineamientos de estructuración de información formulados por (Ortegón, Pacheco, Prieto, 2005; SENPLADES, 2014). La recolección de datos se realizó en los sitios de muestreo: prácticas productivas ancestrales (X 740501, Y 983045, Z 3838 msnm), páramo herbáceo (X 739300 Y 
9832200), páramo seco (X 741670 Y 9832610), microcuenca ( $X 740975$ Y 9830354), y gelidofitia (X 739805 Y 9837044). La identificación de estrategias se efectuó en talleres participativos con los habitantes de la comunidad Pulinguí San Pablo y Chorrera Mirador. Los resultados se presentaron en diagramas de elementos de planificación estratégica.

\section{Resultados}

\subsection{Caracterización del socio- ecosistema del páramo}

En cuanto al ámbito socio- cultural, las comunidades Pulinguí San Pablo y Chorrera Mirador, conformadas por 186 habitantes, están autoidentificadas como indígenas y pertenecientes al pueblo Puruhá, evidenciándose una población mayoritariamente joven de entre 15 y 19 años (INEC, 2010). La principales fuentes de ingresos para estas comunidades son la ganadería, agricultura y turismo, lo cual genera niveles de migración equivalente a un 15,6\% (18 casos de migración), y 1,5\% en migración externa (2 casos de migración a Estados Unidos). Estas comunidades tienen acceso a servicios básicos como energía eléctrica y agua entubada. Además cuentan con servicios de comunicación como radio y televisión y accesibilidad.

En cuanto a lo ambiental, Pulinguí San Pablo y Chorrera Mirador se ubican sobre los 3000 msnm, posee una temperatura de $6^{\circ} \mathrm{C}$ y una precipitación anual de $1000 \mathrm{~mm}$ (GAD Parroquia-Rural de San Juan, 2011). Este sector posee dos estaciones: lluviosa (entre enero y abril alcanzando una precipitación de $1000 \mathrm{~mm} / \mathrm{año}$ ) y seca (entre mayo y septiembre), presentándose heladas constantemente ya que los vientos son fuertes. Los suelos son de origen volcánico, (son negros, profundos y de textura media) en los cuales se observa una topografía irregular con pendientes que sobrepasan el 50\%, destacando el Nevado Chimborazo (6.310 msnm). Las comunidades tienen una extensión de 4567,20 ha. de las que el 97,84\% corresponde a zonas de páramo (4468,7 ha), el 2,06\% se caracteriza por ser pastos y cultivos $(93,9 \mathrm{ha})$, mientras que el $0,10 \%$ son zonas pobladas (4,6 ha). De acuerdo con Sierra, (1999) este ecosistema se divide en tres tipos de vegetación: 1) Herbáceo (se localiza entre los 3400 - 4300 msnm, con presencia de vegetación herbácea como Azorella, Calamagostris); 2) Seco (se ubica entre los 3500 - 4200 msnm, y está conformado por especies de Chuquiraga, Senecio y Plantago spp); 3) Gelidofitia (se localiza sobre los $4700 \mathrm{msnm}$ y es una zona anexa a las nieves perpetuas con presencia de algunos líquenes).

Específicamente, la cobertura vegetal está representada por páramo herbáceo $(37,8 \%)$, páramo seco y zonas de ríos $(32,2 \%)$, gelidofitia $(19,0 \%)$ y finalmente zonas de producción (11\%) en las cuales las especies de Bidens andicola, Tipillo sp. Plantago australis, Chuquiraga jussieui (37,0\%) son aprovechadas para la medicina; las especies de Andreana sp, Grimmia sp, Lycopodium 
clavatum L. (22,0\%) tienen un uso turístico; las especies de Hypochaeris sessilifolia, Hypochaeris sessiliflora son utilizadas como alimentación para animales (19,4\%); las especies de Solanum tuberosum, Vicia faba, Ordeum vulgare, Ullucus tuberosus(13,6\%) sirven para alimentación de pobladores, y las especies Calamagrostis intermedia, Hipericum juniperium (8\%) se usan como leña. Se ha registrado además la presencia de 18 especies de fauna de las cuales, el $72 \%$ se localizan en el páramo herbáceo y el $28 \%$ en el páramo seco, las mismas que son aprovechadas por el turismo en un $89 \%$ (siendo las más representativas Lesbia victorae, Pyrocephalus rubinus, Buteo magnirostris, Oreotrochilus chimborazo, Turdus serranus), y el 11\% para alimentación de pobladores (Lama glama, Silvylagus brasilensis).

En cuanto al suministro de servicios ecosistémicos en el sector se describen los siguientes: 1) Aprovisionamiento: el principal servicio es el agua, con su red hidrológica del río Chimborazo formado a partir del río Pulinguí y vertientes de la Chorrera, misma que tienen una capacidad de 6 litros/s y presenta un análisis físico- químico estable, ya que tiene un $\mathrm{pH}$ neutro $(6,7)$, una conductividad eléctrica no salina (311) y potasio bajo $(2,5)$, encontrándose de esta forma dentro de los rangos permisibles para regadío. Además Pulinguí San Pablo y Chorrera Mirador en sus diferentes tipos de vegetaciones tienen una capacidad de retención de 7’101.064,10 metros cúbicos de agua (GAD Provincial de Chimborazo, 2014). 2) Regulación: el principal servicio es la regulación hídrica, ya que existe una capacidad de agua por unidad de volumen $(80-90 \%$ por cm3) (Ministerio del Ambiente, 2012), gracias a factores como humedad, temperatura y concentración de carbono orgánico en el suelo. 3) Culturales: los principales servicios son: a.- estético, el sector cuenta con atractivos turísticos como el Bosque de Polylepis, Templo Machay (ubicado a 4668msnm), Cascada La Chorrera (ubicado a $3720 \mathrm{msnm}$, tiene una altura de hasta 100m), Nevado Chimborazo (6268msnm) donde se combina la belleza del paisaje, su biodiversidad y la cultura; b.- turismo, ya que en el lugar se desarrollan las modalidades de turismo comunitario y de aventura.

En cuanto al ámbito económico, las principales actividades del sector son: a) Agricultura, generalmente se cultiva papa y haba, (utilizados para el autoconsumo) para el cual se emplea tractor, además agroquímicos, por lo cual el uso de abonos orgánicos no está muy generalizado entre la población; además no existe un sistema en el cual se pueda aprovechar los desechos orgánicos en los cultivos. b) Ganadería, estas comunidades desde hace pocos años están emprendiendo la producción de camélidos andinos (Lama glama, Lama pacos) como parte del Programa Reintroducción de Camélidos Sudamericanos impulsado por el Ministerio del Ambiente. Los individuos han sido destinados para incrementar la población de estas especies contando con un total de 20 individuos en la comunidad Pulinguí San Pablo y de 150 individuos en la comunidad Chorrera Mirador. c) Turismo Comunitario, modalidad que en el año 2013 recibió un flujo de 305 turistas, quienes fueron atendidos en el Centro de Turismo Casa Cóndor, el cual dispone de 30 
plazas, servicios de hospedaje alimentación, transporte interno con 10 caballos (Corporación de Turismo Comunitario de Chimborazo, 2012), el mismo que aprovecha 4 atractivos (3 de categoría sitio natural y 1 de categoría manifestación cultural). Adicionalmente en la zona se desarrolla turismo de aventura a través de la atención a turistas en el Mountain Lodge Estrella del Chimborazo, el cual cuenta con 16 plazas, y servicios de hospedaje y alimentación. Además está presente la operadora de turismo Puruhá Razurku (que se dedica a la prestación de servicios turísticos en la zona).

En cuanto al ámbito político- administrativo, históricamente sus tierras son comunitarias; en la época de las haciendas (año 1960) este territorio fue propiedad de la hacienda García y con el surgimiento de la expropiación de las mismas (año 1980), estos predios pasaron a ser propiedad de los comuneros - habitantes del sector. Con el tiempo y el incremento de la población surge la necesidad de producir y generar recursos alimenticios y económicos, por lo tanto, se transformaron en tierras individuales para constituirse en sistemas de herencias ancestrales y a la vez en sistemas mercantiles (compra-venta de los predios). El total del territorio es de 4567,2 ha, de estas, el $2,2 \%$ son zonas pobladas y destinadas para pastos y cultivos.

En estas comunidades se practica el pleno derecho a la autonomía y al autogobierno. Los habitantes eligen a las autoridades (presidente, vicepresidente, secretario, tesorero, vocales) en asambleas comunitarias con la participación de todos los pobladores. Las instituciones que trabajan en el sector son 3 de tipo público y 1 de tipo comunitario: Ministerio del Ambiente con capacitaciones en turismo y con el programa Socio-Páramo se impulsa la conservación del páramo (desde el año 2012). El Gobierno Autónomo Descentralizado Provincial de Chimborazo que promueve el manejo y cogestión de la microcuenca del río Chimborazo (desde el año 2013). El Gobierno Autónomo Descentralizado Parroquial Rural San Juan lleva a cabo el programa conservación ambiental (desde el año 2012). La Corporación para el Desarrollo del Turismo Comunitario de Chimborazo que impulsa la implementación de proyectos de turismo comunitario y capacitaciones (desde el año 2009), los mismos que en la actualidad se encuentran vigentes.

\subsection{Determinación de objetos de conservación del socio-ecosistema páramo}

Se identificaron 4 objetos de conservación (Tabla 1), mismos que representan las siguientes características: a) Categoría, 3 son de categoría sitio natural y 1 de categoría manifestación cultural. b) Características físicas, los objetos de conservación se ubican entre los 3748 y 4671 msnm y poseen una temperatura promedio de $5^{\circ} \mathrm{C}$. c) Valoración, los objetos de conservación son sensibles al cambio (2M), razón por la cual se encuentran en proceso de deterioro (2PD); presentan una importancia intrínseca con el $50 \%$ por su conectividad (Prácticas productivas ancestrales, Páramo herbáceo) y con el $50 \%$ por su representatividad (Páramo seco, Microcuenca 
del río Chimborazo). En cuanto a servicios ecosistémicos, el $67 \%$ brinda servicios de suministro (1S), mientras que el 33\% son de regulación (3R). Además, la presencia de fauna es alta (1 A) en el páramo seco, media (2M) en el páramo herbáceo y baja (1B) en la microcuenca. Adicionalmente el uso actual de los objetos de conservación es económico con el $75 \%$ (2E), y turístico (1T) con el 25\%.

Tabla 1. Determinación de objetos de conservación

\begin{tabular}{|c|c|c|}
\hline Filtro grueso & Filtro fino & Descripción \\
\hline $\begin{array}{l}\text { Prácticas } \\
\text { productivas } \\
\text { tradicionales }\end{array}$ & $\begin{array}{l}\text {-Técnicas tradicionales } \\
\text {-Formas de siembra y } \\
\text { cosecha } \\
\text {-Conocimientos y } \\
\text { tradiciones andinas }\end{array}$ & $\begin{array}{l}\text { Las prácticas productivas tradicionales ayudan a mantener } \\
\text { la conservación y uso sostenible del recurso suelo, por } \\
\text { cuanto tiene relación directa con la selección y preparación } \\
\text { de la semilla, la preparación de la tierra, el uso de } \\
\text { herramientas agrícolas y la aplicación de técnicas de } \\
\text { control de plagas y cosecha. }\end{array}$ \\
\hline $\begin{array}{l}\text { Páramo } \\
\text { herbáceo }\end{array}$ & $\begin{array}{l}\text {-Pajonal (Calamagrostis } \\
\text { intermedia) } \\
\text {-Almohadilla (Azorella } \\
\text { pedunculata) }\end{array}$ & $\begin{array}{l}\text { Es la base para la formación de las microcuencas, además } \\
\text { que mantienen los caudales del sistema hidrológico } \\
\text { (6litros/ segundo) y a la vez permiten el almacenamiento y } \\
\text { liberación de } 7^{\prime} 101.064,10 \text { metros cúbicos/ año de agua } \\
\text { (GAD Provincial de Chimborazo, 2014). }\end{array}$ \\
\hline Pára & $\begin{array}{l}\text {-Colibrí estrella del } \\
\text { Chimborazo } \\
\text { (Oreotrochilus } \\
\text { chimborazo) } \\
\text {-Chuquiragua } \\
\text { (Chuquiraga jussieui) } \\
\text {-Venado (Odocoileus } \\
\text { virginianus) } \\
\text {-Alpaca (Lama pacos) }\end{array}$ & $\begin{array}{l}\text { Permite proteger especies representativas y amenazadas, } \\
\text { las mismas que se citan acontinuación: } \\
\text {-Representativas } \\
\text { Colibrí estrella del Chimborazo (Oreotrochilus chimborazo) } \\
\text { (ECOLAP y MAE, 2007). } \\
\text {-Amenazadas } \\
\text { Vicuña (Vicugna vicugna) Preocupación menor (LC) } \\
\text { (UICN, 2014), Apéndice II (CITES, 2015). } \\
\text { Venado (Odocoileus virginianus) Preocupación menor } \\
\text { (UICN, 2014), Preocupación menor (Tirira, 2011). } \\
\text { Además contribuye a la preservación de los procesos } \\
\text { ecológicos de este ecosistema, así como el uso público y } \\
\text { turismo. }\end{array}$ \\
\hline $\begin{array}{l}\text { Microcuenca } \\
\text { del río } \\
\text { Chimborazo }\end{array}$ & $\begin{array}{l}\text {-Cascada La Chorrera } \\
\text { - Fuentes de agua } \\
\text { asociados a la } \\
\text { microcuenca } \\
\text { - Llantén (Plantago spp) }\end{array}$ & $\begin{array}{l}\text { Ayuda a mantener los caudales de agua. Es una de las } \\
\text { prioridades en las que se enmarcan los gobiernos de turno } \\
\text { como lo establece el art. } 411 \text { de la Constitución de la } \\
\text { República del Ecuador (Asamblea Nacional, 2008), } \\
\text { estableciendo mecanismos integrales y participativos de } \\
\text { conservación, preservación, manejo sustentable, } \\
\text { restauración del recurso hídrico para aumentar la eficiencia } \\
\text { en el uso de las fuentes hídricas para que la población } \\
\text { pueda acceder a un recurso de calidad como lo establece } \\
\text { el objetivo 7-numeral } 6 \text { "Políticas y Lineamientos } \\
\text { Estratégicos" del Plan del Buen Vivir (SENPLADES, 2013- } \\
\text { 2017). }\end{array}$ \\
\hline
\end{tabular}

Estos objetos de conservación calificados en torno a los parámetros de tamaño, condición y contexto paisajístico, arrojaron como resultado un promedio general de 3,3 (Tabla 2), lo cual significa que la viabilidad es "Buena", es decir, que los objetos identificados se encuentran en proceso de deterioro; sin embargo sus impactos se los puede minimizar con acciones adecuadas y con el compromiso y participación activa de los involucrados, ya que estos objetos ejercen 
influencia directa sobre el funcionamiento del socioecosistema, el flujo de servicios ecosistémicos y por ende el bienestar humano de sus beneficiarios.

Tabla 2. Viabilidad de objetos de conservación

\begin{tabular}{|l|c|c|c|c|c|}
\hline $\begin{array}{l}\text { Objetos focales de } \\
\text { manejo }\end{array}$ & Tamaño & Condición & $\begin{array}{c}\text { Contexto } \\
\text { paisajístico }\end{array}$ & $\begin{array}{c}\text { Valor jerárquico } \\
\text { global de } \\
\text { viabilidad }\end{array}$ & Ponderación \\
\hline $\begin{array}{l}\text { Prácticas } \\
\text { ancestrales }\end{array}$ & $4,0(\mathrm{MB})$ & $2,5(\mathrm{R})$ & $2,5(\mathrm{R})$ & 9,0 & $3,0(\mathrm{Bueno})$ \\
\hline Páramo herbáceo & $3,5(\mathrm{~B})$ & $2,5(\mathrm{R})$ & $3,5(\mathrm{~B})$ & 9,5 & $3,2(\mathrm{Bueno})$ \\
\hline Páramo seco & $4,0(\mathrm{MB})$ & $2,5(\mathrm{R})$ & $3,5(\mathrm{~B})$ & 10,0 & $3,3($ Bueno) \\
\hline $\begin{array}{l}\text { Microcuenca del } \\
\text { Río Chimborazo }\end{array}$ & $2,5(\mathrm{R})$ & $4,0(\mathrm{MB})$ & $4,0(\mathrm{MB})$ & 10,5 & $3,5($ Bueno) \\
\hline \multicolumn{2}{|l|}{ CALIFICACIÓN GLOBAL DE LOS OBJETOS FOCALES } & 13,0 & 3,3 (Bueno) \\
\hline
\end{tabular}

\subsection{Formulación de estrategias de conservación del socio-ecosistema páramo}

Ante las amenazas identificadas en el socio-ecosistema, la aplicación de estrategias contribuirá al proceso de conservación y uso sustentable del páramo; adicionalmente, permitirá que los actores que tienen competencia en la gestión del páramo sumen esfuerzos.

\subsubsection{Estrategias de conservación del objeto "prácticas productivas tradicionales"}

La pérdida de la tradición y la desvalorización cultural son las presiones que degradan de forma moderada el recurso (ponderación de 2,5=Medio), las cuales están impulsadas a una escala local por la adopción de nuevas formas de vida y cambios en la forma de subsistencia de los pobladores locales (ponderación de 3,5=Alta). Para manejar las presiones y minimizar las fuentes de presión en el objeto de conservación se plantea como estrategia la "evaluación del componente biocultural del socio ecosistema", misma que contribuirá al conocimiento y revalorización de las prácticas productivas tradicionales en los pobladores locales (Figura 1).

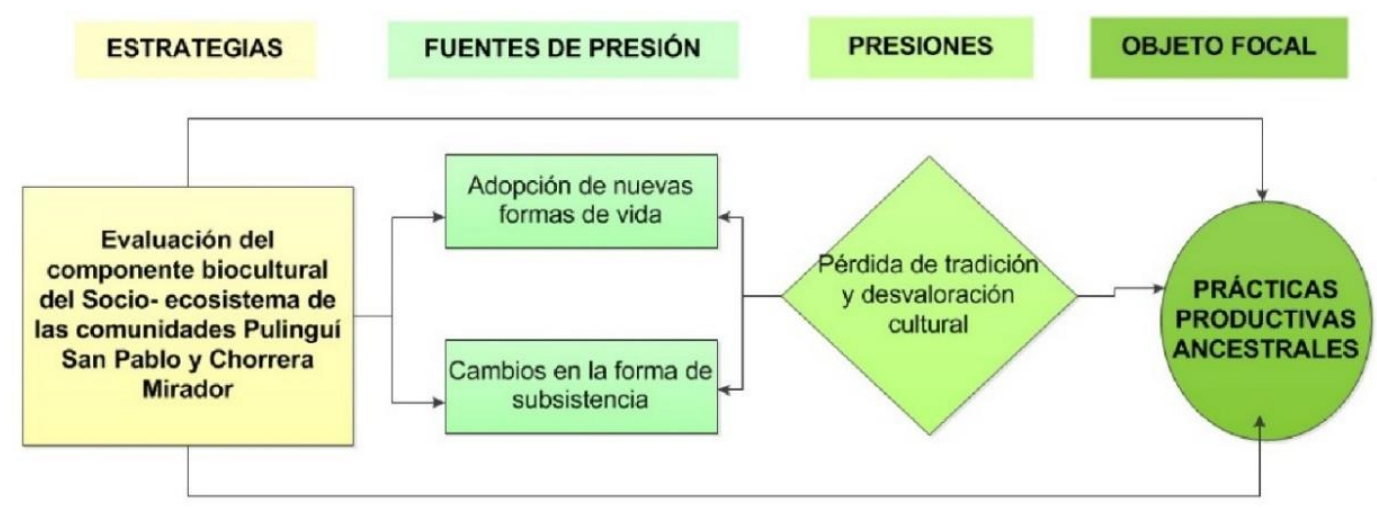

Figura 1. Amenazas-estrategias prácticas productivas tradicionales 


\subsubsection{Estrategias de conservación del objeto "páramo seco"}

La erosión y compactación del suelo son las presiones que degradan de forma severa el recurso (ponderación de $3=A l t a)$, las mismas que están impulsadas a una escala local por la variabilidad climática y el pastoreo de ganado no permitido (ponderación de 2,6=Medio). Para manejar las presiones y minimizar las fuentes de presión del objeto de conservación se plantea como estrategias la "evaluación del estado de conservación del componente biótico del ecosistema páramo", y la "implementación de buenas prácticas de turismo sostenible en las comunidades", las que contribuirán al conocimiento del estado de salud del ecosistema para su conservación y manejo, y a la consolidación de alternativas económicas que permitan minimizar actividades económicas incompatibles con la conservación del páramo (Figura 2).

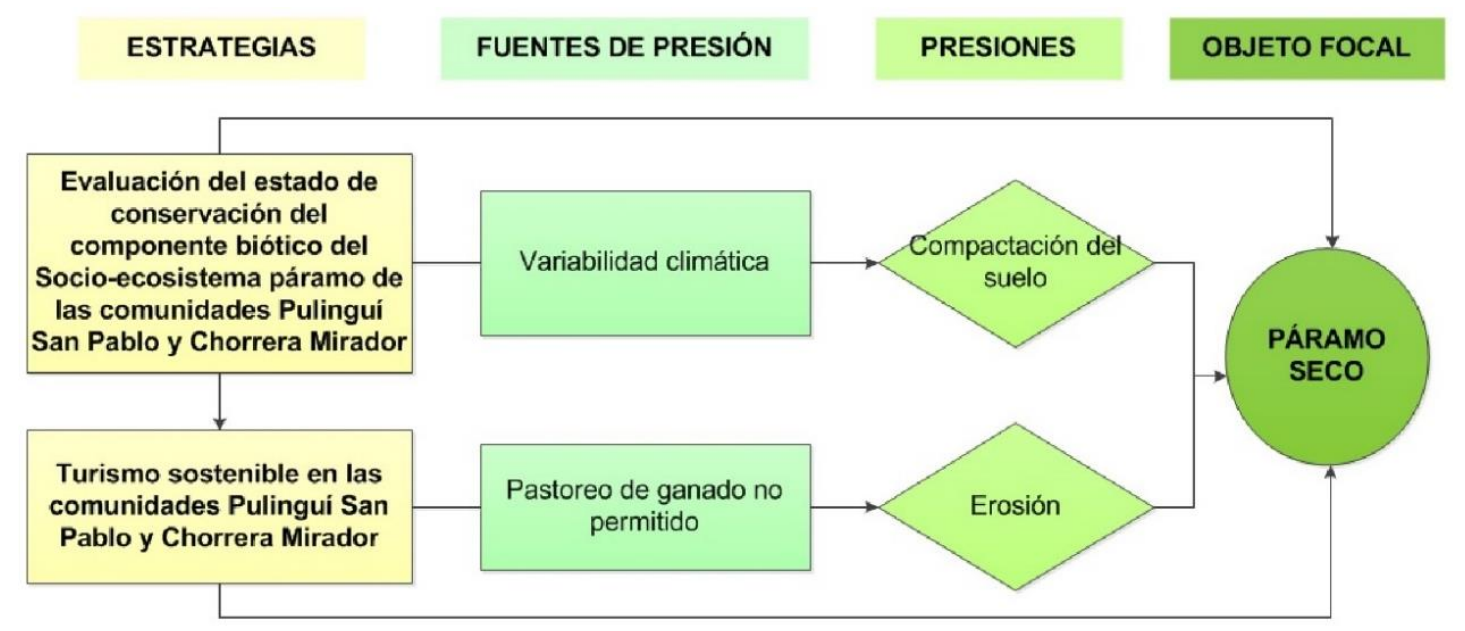

Figura 2. Amenazas-estrategias páramo seco

\subsubsection{Estrategias de manejo del páramo herbáceo}

La quema de páramo, el incremento de la actividad agropecuaria no permitida, y la erosión del suelo son las presiones que degradan de forma severa el recurso (ponderación de 3,5 Alta), las mismas que están impulsadas a una escala local por una cultura socio-económica incompatible con el ecosistema, la creencia de atracción de lluvias, y la aplicación de inadecuadas técnicas agrícolas (ponderación de 3,2=Alta). Para manejar las presiones y minimizar las fuentes de presión del objeto de conservación se plantean como estrategias la "forestación y reforestación de zonas de páramo degradadas", la "implementación de buenas prácticas en la actividad agrícola", y el "mejoramiento de la producción pecuaria", que contribuirán en la restauración ecológica del páramo, al desarrollo económico y seguridad alimentaria de la población local (Figura 3). 


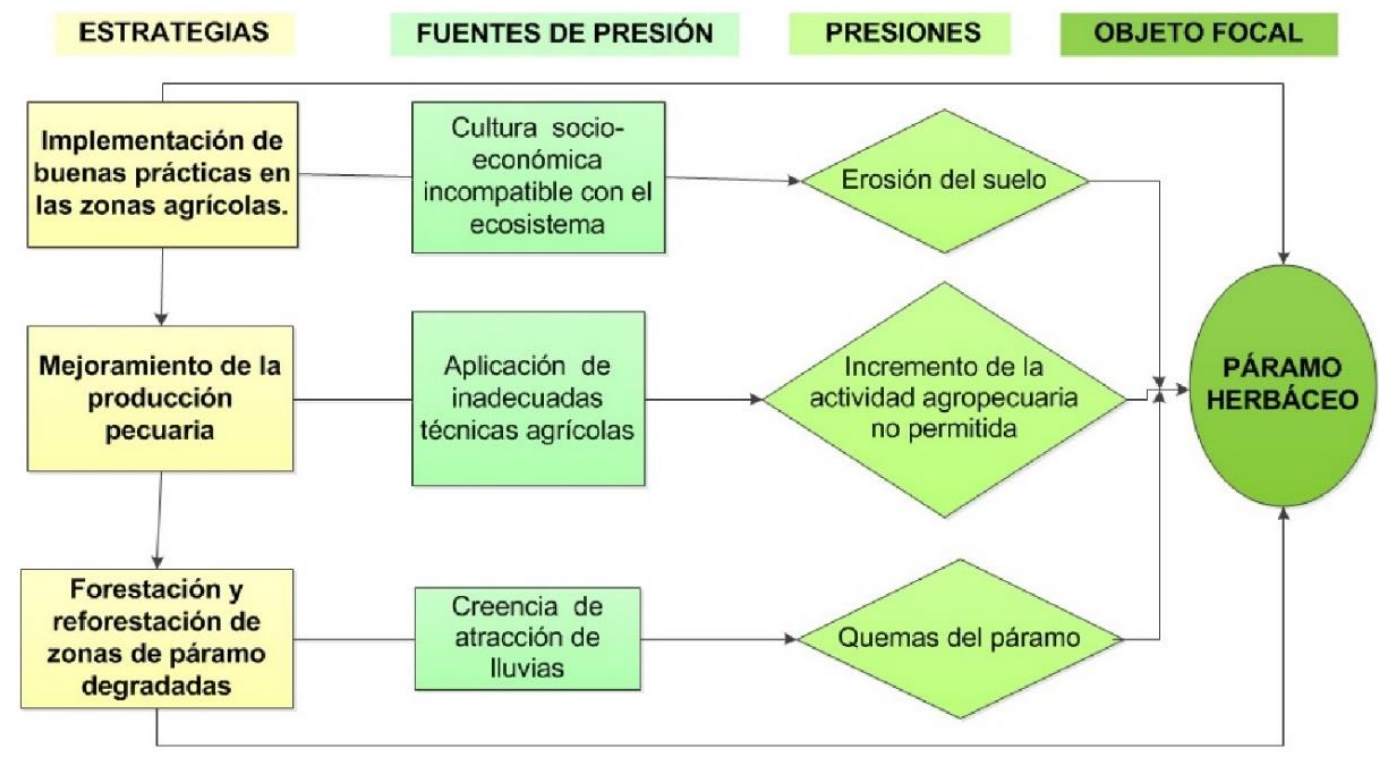

Figura 3. Amenazas-estrategias páramo herbáceo

\subsubsection{Estrategias de manejo de la microcuenca del Río Chimborazo}

La contaminación, la pérdida de vertientes de agua, y los conflictos por el acceso y distribución del agua son las presiones que degradan de forma severa al recurso (ponderación de 3,0=Alta), las mismas que están impulsadas a una escala local por la descarga de fertilizantes y desechos sólidos, quema de pajonales y almohadillas, el pisoteo y sobrecarga de animales en las vertientes, y la canalización de agua (ponderación de 3,2=Alta). Para manejar las presiones y minimizar las fuentes de presión del objeto de conservación se plantean como estrategias la "aplicación de buenas prácticas para la siembra y el uso del agua y el "fortalecimiento organizacional del comité de cogestión del agua", las mismas que contribuirán en el incremento del valor hidrológico del páramo y en el mejoramiento de la relaciones entre las comunidades para la conservación y uso del agua (Figura 4).

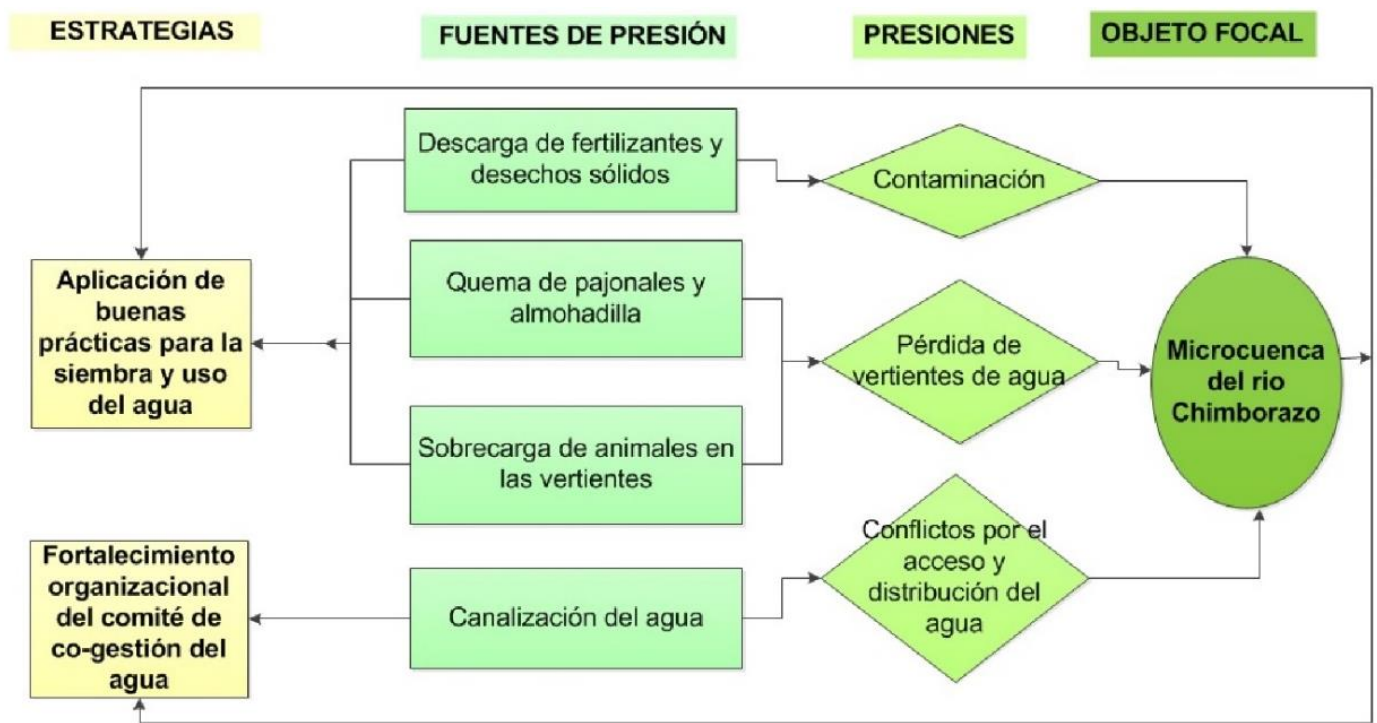

Figura 4. Amenazas-estrategias microcuenca del Río Chimborazo 


\section{Discusión}

Los páramos de las comunidades se encuentran entre los 3200 y 4700 msnm, rango altitudinal que concuerda con (Sierra, 1999, Hosftede, et al, 2014, Suárez, 2016). La cobertura vegetal está caracterizada principalmente por páramo herbáceo y seco, rasgos que cualitativamente son similares a estudios realizados por (Ministerio de Ambiente, 2013, y Caranqui, Lozano y Reyes, 2016). Los asentamientos humanos y actividades agropecuarias tienen una extensión de 98,65 ha., es decir, el 2,16\% de 4567,20 ha, rasgo que difiere de la relación propuesta por (Albán, Argüello, \& Bustamante, 2011) en cuanto a las áreas intervenidas en el ecosistema en estado natural en la provincia de Chimborazo. Los principales servicios ecosistémicos que benefician a las comunidades son aprovisionamiento (agua dulce), regulación (hídrica) y culturales (paisaje y turismo), rasgos que parcialmente son similares a los propuestos por (Hosftede \& Mena, 2006, y Hofstede, 2008) en su aproximación a los servicios del ecosistema páramo.

Los objetos de conservación identificados participativamente ( 3 naturales y 1 cultural) para la presente investigación representan los elementos claves del socio-ecosistema, es decir, si se logra intervenir con actuaciones de conservación y uso sustentable en estos, se podría a mediano y largo plazo, mejorar el estado de salud del socio-ecosistema, el suministro de servicios ecosistémicos, y el bienestar humano de las poblaciones locales beneficiarias. Sin embargo, la viabilidad de objetos de conservación reportada en el presente trabajo indica que el socioecosistema se encuentra en proceso de deterioro, por cuanto, existen presiones asociadas a la pérdida de las tradiciones y desvalorización cultural, y al incremento de las actividades agrícolas y pecuarias no permitidas; estos rasgos son parcialmente similares a los identificados por (Albán, Argüello, \& Bustamante, 2011, Ministerio de Ambiente, 2012, Caranqui, Haro, Salas, \& Palacios, 2013, Hosftede, et al, 2014,) en cuanto a las amenazas del ecosistema de páramo, las que se incrementan proporcionalmente al crecimiento de la población local (Mena, Medina, \& Hosftede, 2001).

Las actuaciones de intervención propuestas, en esencia, recogen el enfoque ecosistémico, y cumplen los 12 principios para su aplicación, por cuanto, la identificación de objetos de conservación se lo hizo en un nivel de organización local y desde sus habitantes en una escala espacial y temporal apropiada; las actuaciones propuestas consideran el funcionamiento del socio-ecosistema y pretenden estimular el desarrollo económico de las poblaciones locales a través del equilibrio entre conservación y uso sustentable de los recursos, los efectos de la aplicación de las actuaciones en los ecosistemas asociados al socio-ecosistema serán positivas, y se ha valorado e incluido en las actuaciones propuestas el conocimiento de las poblaciones locales y sectores de la sociedad, tal cual lo recomienda (Shepherd, 2006). Este proceso ha 
permitido formular 8 estrategias, las mismas que son parcialmente similares a las propuestas por (Segovia, 2012), para la adaptación del cambio climático en la provincia de Chimborazo.

\section{Conclusiones y Recomendaciones}

En las comunidades Pulinguí San Pablo y Chorrera Mirador se desarrollan actividades agropecuarias y turísticas, las mismas que reciben el apoyo de entidades gubernamentales, convirtiéndose de esta forma en las principales fuentes de ingresos económicos del sector. Los productos que mayoritariamente se cultivan son: la papa y haba, mientras que la ganadería se encamina a la producción de camélidos andinos (Lama pacos); asimismo las principales modalidades de turismo son de aventura y comunitario, las mismas que reciben un flujo promedio anual de 305 visitantes.

De las 4567,2 ha., que constituyen el socio-ecosistema de las comunidades de Pulinguí San Pablo y Chorrera Mirador, el 97,84\% corresponde a zonas de páramo (4468,7 ha), el cual está conformado por 3 tipos de vegetación: herbáceo, seco, gelidofitia. En este ecosistema se puede apreciar un total de 18 especies de fauna, aprovechadas el $89 \%$ por el turismo y el $11 \%$ para alimentación de los pobladores, además se han registrado un total de 36 especies de flora utilizadas el $22,2 \%$ en la medicina, el $19,4 \%$ en el turismo, el 13,9\% para alimentación de los pobladores y el $8 \%$ se utiliza como leña. Adicionalmente el principal servicio ecosistémico es la regulación hídrica y el suministro de agua, ya que este ecosistema tiene una capacidad de retención de 7’101.064,10 metros cúbicos por año.

Para conocer el estado del socio-ecosistema del páramo de las comunidades de Pulinguí San Pablo y Chorrera Mirador se seleccionaron 4 objetos focales, 3 de categoría sitio natural y 1 de categoría manifestación cultural, los cuales se encuentran en proceso de deterioro; sin embargo, ambientalmente son viables con una calificación de 3,3 (Buena), es decir que su sensibilidad al cambio es media (2M), su conectividad y representatividad presentan una importancia del $50 \%$. Adicionalmente, el uso actual de los objetos focales es económico (75\%) y turístico (25\%).

Las presiones a los objetos focales arrojaron un promedio de 3,2 Alto, es decir que la presión afecta al recurso en muchos sectores y el resultado de las fuentes de presión es de 3,4 Alto, lo cual indica que el contribuyente es alto, pero sus efectos son reversibles. Para minimizar estos efectos se plantearon 8 estrategias encaminadas a la conservación y aprovechamiento de cada uno de los objetos focales, ya que los impactos ocasionados se los puede revertir con la implementación de actuaciones construidas con los actores. 


\section{Bibliografía}

Anderies, J.M., Janssen, M.A., \& Ostrom, E. (2004). A framework to analyze the robustness of social-ecological systems from an institutional pesrpective. Conservation Ecology 9, 18. http://www.ecologyandsociety.org/

Albán, M., Argüello, M., \& Bustamante, M. (2011). Los páramos de Chimborazo. Un estudio socio ambiental para la toma de decisiones. Quito: gobierno autónomo descentralizado de Chimborazo/EcoCiencia/CODESAN/Programa BioAndes/Proyecto Páramo Andino.

Armas, A. (2014). Elaboración del plan de conservación para el aprovechamiento turístico del paisaje del ecosistema páramo de las comunidades Pulinguí San Pablo y Chorrera Mirador. Riobamba: ESPOCH.

Asamblea Nacional. (2008). La Constitución. Montecristi: SENPLADES.

Beltran, K. (2010). Áreas prioritarias para conservación de páramos en la provincia de Chimborazo. Quito: EcoCiencia y Condesan.

Camacho, V., Ruiz, A. (2011). Marco Conceptual y conservación de los servicios ecosistémicos. Revista Bio Ciencias, Vol.1, Nro 4, Pág. 3 -15.

Caranqui, J., Haro, W., Salas, F., \& Palacios, C. (2013). Diversidad y similitud en los páramos del Chimborazo. Riobamba: Herbario Escuela Superior Politécnica de Chimborazo/Gobierno Autónomo Descentraliado de Chimborazo.

Caranqui, J., Lozano, P., Reyes, J. (2016). Composición y diversidad florística de los páramos en la Reserva de Producción de Fauna Chimborazo, Ecuador. Revista Enfoque UTE, V.7-N1 pp. 33-45.

Convención sobre el Comercio Internacional de Especies Amenazadas de Fauna y Flora Silvestres (CITES). (2015). Apéndices I, II y III. Quito- Ecuador.

Consorcio de Gobiernos Parroquiales Rurales del Ecuador (CONOGOPARE) (2011). Planes de Desarrollo y Ordenamiento Territorial. Riobamba- Ecuador: SENPLADES.

Consorcio de Gobiernos Parroquiales Rurales del Ecuador (CONOGOPARE) (2011). Sistema de Información Geográfica. Riobamba-Ecuador.SENPLADES.

Corporación para el Desarrollo del Turismo Comunitario de Chimborazo (CORDTUCH) (2012). Turismo comunitario. Riobamba- Ecuador.

Gobierno Autónomo Descentralizado Provincial (GADPCH). (2014). Plan de Manejo y Cogestión de la Microcuenca del Rio Chimborazo. Riobamba- Ecuador.

Gobierno Autónomo Desentralizado Parroquial Rural San Juan (GADPRSJ). (2011). Plan de Desarrollo y Ordenamiento Territorial de la parroquia San Juan. Riobamba- Ecuador: SENPLADES.

Geilfus, F. (1997). 80 Herramientas para el Desarrollo Participativo: diagnóstico, planificación, monitoreo, evaluación. San Salvador- El Salvador: Prochalate-IICA. 
Granizo, T., Molina, M. E., Secaira, E., Herrera, B., Benítez , S., Maldonado, O., y otros. (2006). Manual de Planificación para la Conservación de Áreas PCA. Quito: TNC y USAID.

Hosftede, R. (2008). Los servicios del ecosistema páramo: Una visión desde la Evaluación de ecosistemas del Milenio. Quito: EcoCiencia.

Hosftede, R., \& Mena, P. (2006). Los Páramos Ecuatorianos. Quito: EcoCiencia.

Hosftede, R., Calles, J., López, V., Polanco, R., Torres, F., Ulloa, J., y otros. (2014). Los Páramos Andinos ¿Qué sabemos? Estado de conocimiento sobre el impacto del cambio climático en el ecosistema páramo. Quito: UICN Quito.

Instituto Nacional de Estadísticas y Censos (INEC). (2010). Censo de población y vivienda. QuitoEcuador: INEC, SENPLADES.

Instituto de Ecología Aplicada y Ministerio del Ambiente (ECOLAP y MAE). (2007). Guía del Patrimonio de Áreas Naturales Protegidas del Ecuador- RPFCH. Quito- Ecuador: ECOFUND, FAN, DarwinNet, IGM.

Martin, B., Gómez, E., Montes, C. (2009). "Un marco conceptual para la gestión de las interacciones naturaleza sociedad en un mundo cambiante". En: Cuaderno Interdisciplinar de Desarrollo Sostenible. Número 3, pág: 229 - 258. España.

Mena V, P. (2008). El estado de salud de los páramos en el Ecuador: Alternativas interesantes y pronósticos complicados. Quito: Proyecto Páramo Andino/ EcoCiencia.

Mena, P. (s.f.). El estado de salud de los páramos en el Ecuador: Alternativas interesantes y pronósticos complicados. Quito: Proyecto Páramo Andino/ EcoCiencia.

Mena, P.(b), \& Hosftede, R. (2006). Los páramos Ecuatorianos. Quito: EcoCiencia.

Mena, P.(c), Medina, G., \& Hosftede, R. (2001). Los Páramos del Ecuador, Particularidades, Problemas, Perspectivas. Quito: Abya Yala - Proyecto Páramo.

Ministerio del Ambiente (MAE). (2012). Propuesta preliminar de un sistema de clasificación de vegetación para el Ecuador Continental. Quito- Ecuador: Proyecto INEFAN/ GEF- BIRF y Ecociencia.

Ministerio del Ambiente (MAE). (2014). Plan Operativo Anual con base en elementos de Planificación Estratégica de Manejo- RPFCH. Puembo- Ecuador.

Millenium Ecosystem Assessment. (2003). Ecosystems and human well-being: $A$ framework for assessment, Island press, Washington. DC.

Montes, C., \& Sala, O. (2007). La Evaluación de los Ecosistemas del Milenio. Las relaciones entre el funcionamiento de los ecosistemas y el bienestar humano. Revista Ecosistemas 16 (3): 137-147.

Ortegón, E., Pacheco, J. F., \& Prieto, A. (2005). Metodología del marco lógico para la planificación, el seguimiento y la evaluación de programas y proyectos. Santiago de Chile: ILPES. 
Secretaría Nacional de Planificación y Desarrollo (SENPLADES). (2011). Guía de contenidos y procesos para la formulación de Planes de Desarrollo y Ordenamiento Territorial de provincias,cantones y parroquias. Quito- Ecuador: SENPLADES.

Secretaría Nacional de Planificación y Desarrollo (SENPLADES). (2013- 2017). Plan Nacional del Buen Vivir. Quito- Ecuador: SENPLADES.

Secretaría Nacional de Planificación y Desarrollo (SENPLADES) (2014). Formato para la presentación de perfiles de proyecto. Quito- Ecuador: SENPLADES.

Shepherd, G. (2006). El Enfoque Ecosistémico: Cinco Pasos para su Implementación. UICN, Gland, Suiza y Cambridge, Reino Unido. 1 pp.

Segovia, F. (2012). El clima cambia. Cambia tú también. Adaptación al cambio climático en comunidades del Chimborazo. UICN. Biblioteca Nacional de Perú.

Sierra, R. (1999). Propuesta preliminar de un sistema de clasificación de vegetación para el Ecuador Continental. Quito- Ecuador: Proyecto INEFAN/GEF-BIR.

Suárez, L. (2016). CODESAN. Recuperado el 14 de 02 de 2016, de Los Páramos como Paisajes Culturales en el Ecuador:

http://www.condesan.org/unesco/Cap\%2012\%20Ecuador\%20Luis\%20Suarez.pdf

The Nature Conservancy (TNC). (2005). Conservación para planificación de áreas, . Quito: Fundación Natura.

The Nature Conservancy (TNC). (2002). Esquema de las Cinco S para la Conservación- Manual de Planificación para la Conservación de Sitios y la Medición del Éxito en Conservación. 2 ed. Fundación Natura.

Tirira, D. (2011). Lista Roja de mamíferos del Ecuador. Quito: Fundación Ecociencia.

Unión Internacional para la Conservación de la Naturaleza (UICN). (2014). Lista Roja de Especies Amenazadas. Recuperado el 2015 de agosto de 30, de http://www.iucnredlist.org/ 